\title{
Desarrollo de un addin para Monodevelop 2.8 que permita la implementación del servicio de suscripciones de ASP.NET 2.0 empleando PostgreSQL 8.4 como gestor de base de datos
}

\author{
Denis Leopoldo Espinoza Hernández \\ Departamento de Computación, Facultad de Ciencias y Tecnologías, Universidad Nacional Autónoma de Nicaragua, \\ León (UNAN-León). \\ Email: denisjev@ct.unanleon.edu.ni
}

Recibido: 15 Septiembre, 2014

Aceptado: Octubre 15, 2014

\section{RESUMEN}

MonoDevelop es un IDE libre y gratuito diseñado como alternativa a Visual Studio de Microsoft para el desarrollo de aplicaciones en $\mathrm{C \#}$ y otros lenguajes soportados por .NET, que pesar de su amplio desarrollo aún adolece de algunas funcionalidades que Visual Studio ofrece. Entre las carencias que MonoDevelop tiene se encuentra el no poseer una interfaz que facilite la configuración y administración del servicios de suscripciones de ASP.NET a como lo tiene Visual Studio lo que obliga al usuario de MonoDevelop a realizar todas las configuraciones de forma manual. En este artículo se describen las etapas realizadas para el desarrollo de un addin (complemento) para MonoDevelop 2.8 que permite la implementación del servicio de suscripciones de ASP.NET 2.0 de manera similar a como se realiza en Visual Studio 2008. Como alternativa al gestor SQL Server 2008 de Microsoft empleado en la implementación del servicio de suscripciones desde Visual Studio 2008, se ha seleccionado PostgreSQL 8.4 como gestor de bases de datos para esta implementación por ser libre, gratuito y de alta compatibilidad con MonoDevelop 2.8.

\section{PALABRAS CLAVES}

Proyecto Mono, MonoDevelop, Servicio de subscripciones, ASP .NET 


\section{1- INTRODUCCIÓN}

El servicio de suscripciones de ASP.NET permite almacenar las credenciales de un usuario que al ser empleadas junto con los controles de inicio de sesión, facilitan la creación de un completo sistema de autenticación para las aplicaciones web. Este servicio emplea por defecto SQL Server como proveedor de datos sin embargo .NET permite la utilización de otros gestores a través de la creación de clases que deriven de MembershipProvider y RoleProvider. Entre las implementaciones que encontramos para otros gestores cabe destacar la hecha por Andri. Web para PostgreSQL 8.4 la cual fue empleada como base en la realización de este trabajo. Microsoft Visual Studio 2008 es un IDE para Windows que facilita a los desarrolladores de aplicaciones web la administración del servicio de suscripciones de ASP.NET 2.0 a través de una herramienta gráfica que permite gestionar todo lo referente a las cuentas de usuarios, funciones y reglas de acceso de la aplicación.

Como alternativas libres y gratuitas a .Net Framework y al IDE Visual Studio 2008 de Microsoft tenemos en primer lugar el proyecto Mono, que permite crear aplicaciones multiplataforma compatibles con Microsoft .Net Framework y en segundo lugar, el IDE MonoDevelop 2.8 que permite a los desarrolladores escribir rápidamente aplicaciones sobre Linux, Windows y Mac OSX. Aunque la plataforma Mono 2.10 soporta el servicio de suscripciones de ASP.NET 2.0, MonoDevelop 2.8 no aporta ninguna funcionalidad para facilitar la configuración y administración de este servicio obligando al desarrollador del sitio a realizar todas las operaciones y configuraciones de forma manual lo cual es un proceso largo y que requiere de muchos conocimientos que no son necesarios cuando se desea realizar el mismo trabajo sobre Visual Studio 2008.

En base a este problema y empleando una de las principales ventajas de MonoDevelop que es el permitir a los desarrolladores añadir nuevas funcionalidades a través de la creación de extensiones mejor conocidas como addins, se decidió realizar un addin para MonoDevelop 2.8 que permita implementar el servicio de suscripciones en las aplicaciones ASP.NET de forma parecida a como este servicio se implementa en Visual Studio 2008, empleando PostgreSQL 8.4 para el almacenamiento de la información de los usuarios y los roles de la aplicación. Este addin permite al desarrollador:

- Seleccionar la base de datos de PostgreSQL a emplear.

- Realizar las modificaciones necesarias en el proyecto de forma automática.

- Visualizar una herramienta web que permitirá administrar los usuarios, roles y reglas de acceso a las diferentes carpetas de la aplicación. 


\section{2- REQUISITOS DELSISTEMA}

Una vez que se realizó el análisis del funcionamiento de la herramienta que Visual Studio 2008 provee para la gestión del servicio de subscripciones y empleando el modelo de casos de uso para la captura de requisitos de usuario se encontraron un total de 10 casos de uso que engloban todas las operaciones que debe cumplir el addins y las cuales se ven reflejadas en la Figura 1 .

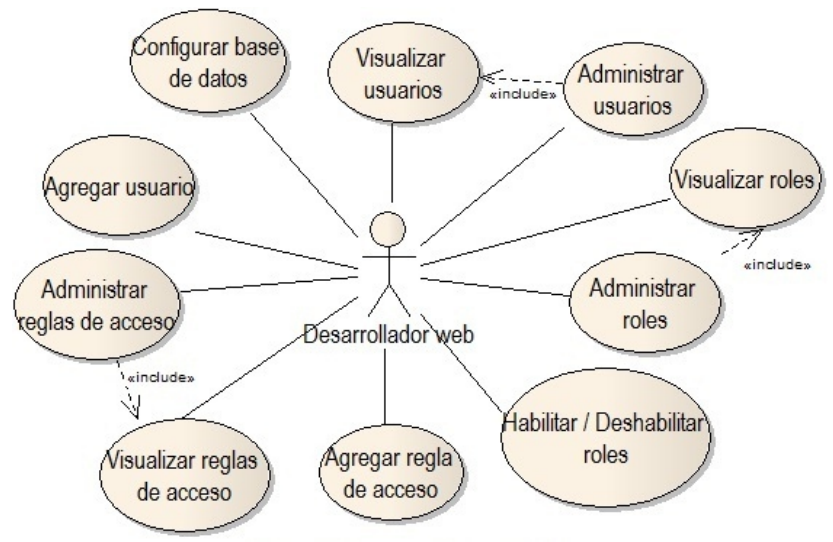

Figura 1. Diagrama de casos de uso

El único actor del sistema es el Desarrollador Web que es todo usuario que desarrolle aplicaciones ASP.NET en MonoDevelop 2.8 y que desee agregar a su proyecto el servicio de suscripciones.

\section{3- DISEÑO DELADDINS}

Una vez extraídos los casos de uso que englobaban toda la funcionalidad que debía proveer el addins, se decidió que estas fueran accedidas a través de dos opciones a las que el desarrollador tuviese acceso desde un submenú llamado Subscription Settings dentro del menú Project del entorno de MonoDevelop 2.8. Las dos opciones son:

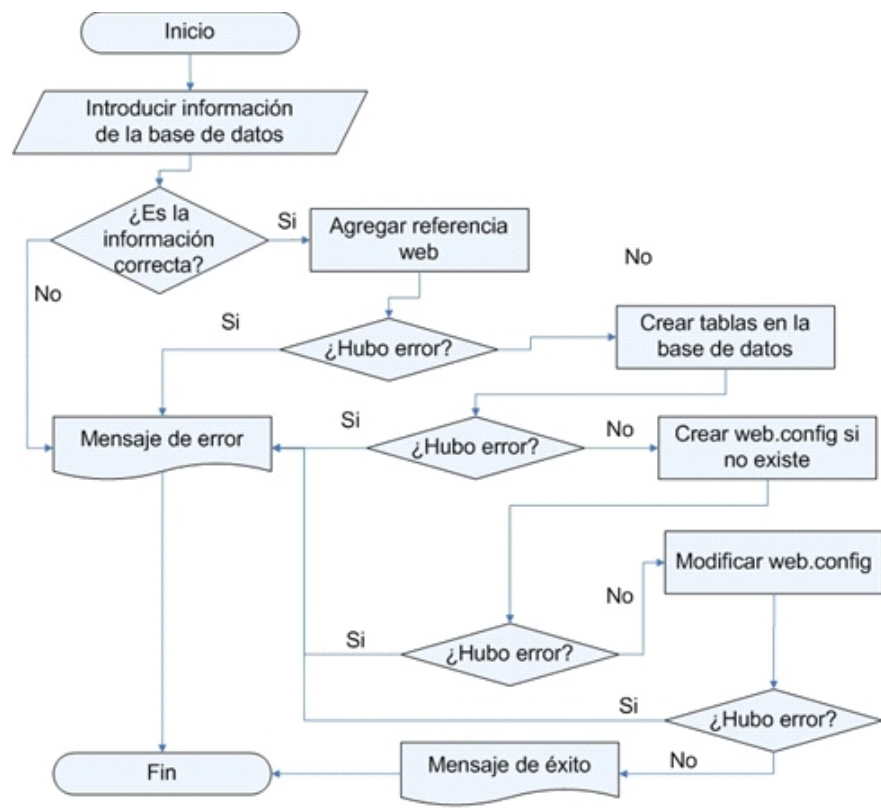

Figura 2. Diagrama de flujo de la opción Database Settings del addin 
La Figura 2 muestra un flujograma en el que se reflejan las operaciones que realiza esta opción. Al acceder a ella, el desarrollador indica la base de datos que desea emplear para el almacenamiento de la información de los usuarios y roles; con esta información el addins crea la estructura de tablas necesaria en la base de datos y posteriormente agrega y/o modifica el web.config de la aplicación para habilitar y configurar el servicio de subscripciones de ASP.NET tomando en cuenta la base de datos antes indicada. Esta opción del addins permite cumplir el caso de uso Configurar base de datos que se muestra en la Figura 1 .

\section{SUBSCRIPTION SETTINGS}

Aunque con la primera opción del addin queda configurado el servicio de suscripciones, la segunda opción permite al desarrollador interactuar con este servicio a través de un sitio web creado para la administración de los usuarios, roles y reglas de acceso que se manejan en la aplicación.

Como se puede observar en la Figura 3 cuando el desarrollador selecciona esta opción se hacen las validaciones correspondientes para lanzar una instancia del servidor XSP2 (servidor de aplicaciones ASP.NET para Linux) el cual carga el sitio web que permite la configuración del servicio de suscripciones para la aplicación que actualmente se esté desarrollando. Se puede lanzar una instancia del servidor XSP2 por cada instancia de MonoDevelop 2.8 que se encuentre ejecutando.

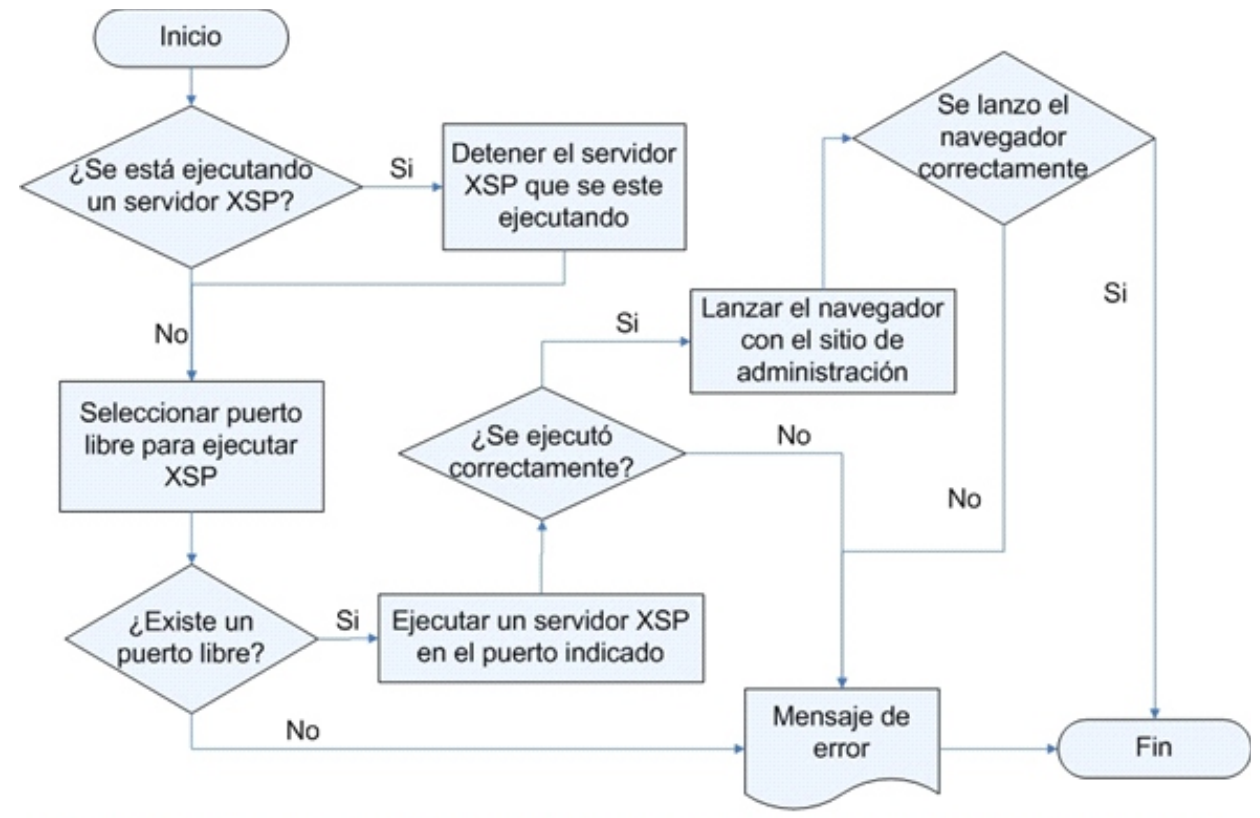

Figura 3. Diagrama de flujo de la opción Subscription Settings del addin

\section{4- DIAGRAMA DE CLASES}

Para lograr las funcionalidades que muestran los casos de uso del addin se han agrupado las clases necesarias en cinco paquetes los cuales se reflejan en la Figura 5.

El paquete SubscriptionServices contiene las clases encargadas de visualizar las opciones del addins dentro de la interfaz de MonoDevelop 2.8. Es en estas clases en las cuales se hace uso de la API de MonoDevelop 2.8 para permitir agregar nuevas opciones al entorno. 


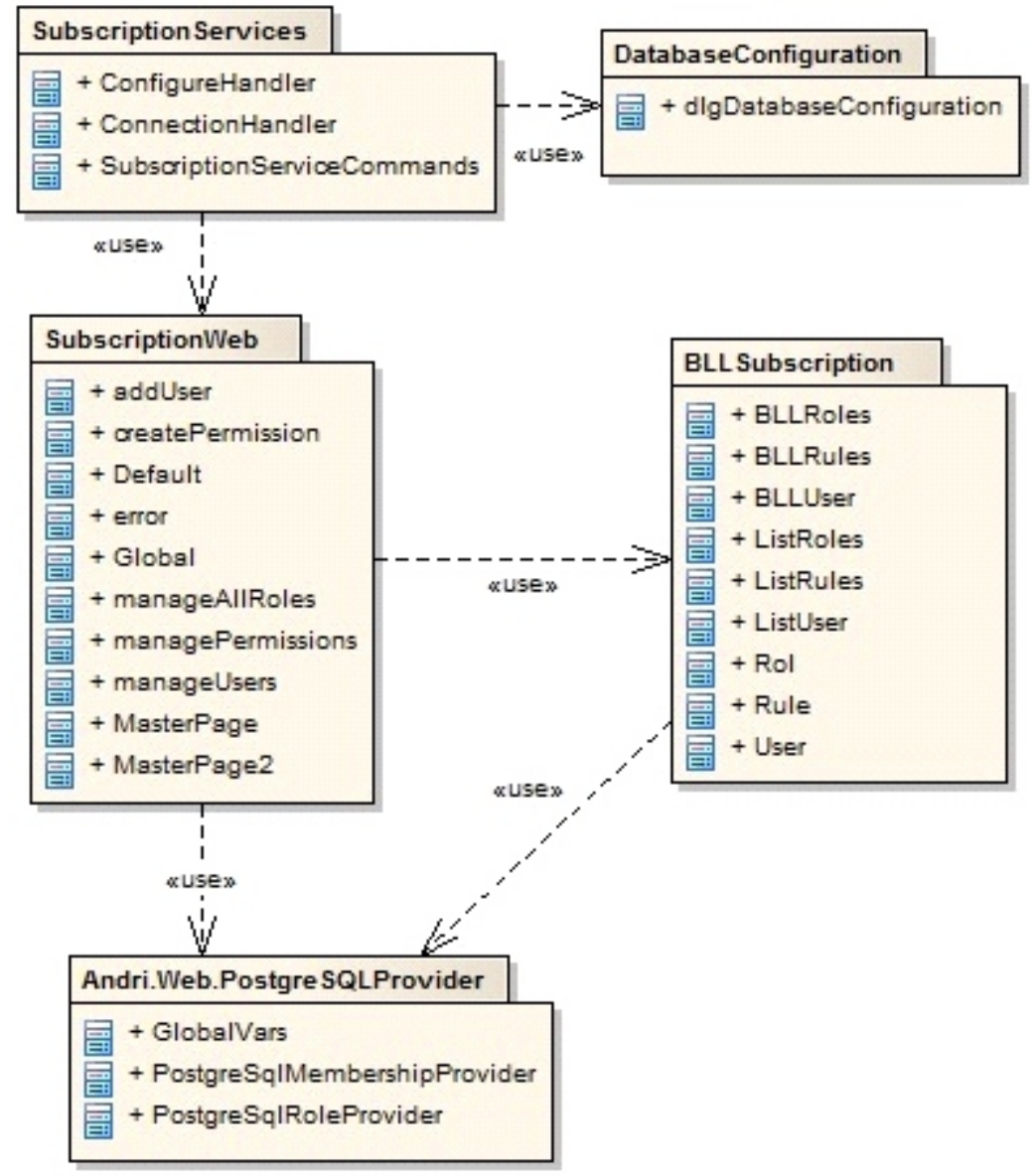

Figura 4. Paquetes de clases de la aplicación

El paquete DatabaseConfiguration posee una única clase llamada dlgDatabaseConfiguration que es la encargada de mostrar una interfaz desarrollada en GTK 2.0 empleada para solicitar la información de la base de datos a emplear por el servicio de suscripciones. La razón por la cual se empleo GTK en lugar de Windows Form fue por mantener la uniformidad en el estilo del entorno pues MonoDevelop 2.8 está desarrollado empleando GTK 2.0.

El paquete Andri.Web.PostgreSQLProvider contiene las clases que implementan el proveedor del servicio de suscripciones para PostgreSQL 8.4. Aunque las clases contenidas en este paquete no han sido desarrolladas por el autor de este artículo y desarrollador del addins, si fueron modificadas por el mismo para lograr las funcionalidades necesarias Estas clases fueron hechas por un desarrollador que se identifica como Andri.Web.

El paquete BLLSubscription contiene las clases de lógica de negocio empleadas para el manejo de usuarios, roles y reglas desde acceso desde la aplicación web.

El paquete SubscriptionWeb encapsula un sitio web que ha sido desarrollado para administrar la aplicación. Este sitio ha sido creado teniendo como modelo el empleado en Visual Studio 2008 con el objetivo de que el usuario se sienta familiarizado con su uso. A como se muestra en la Figura 5 este paquete hace uso del paquete BLLSubscription y Andri.Web.PostgreSQLProvider para el acceso a la base de datos y a los ficheros web.config de la aplicación. 


\section{5- RESULTADOS}

Al finalizar el desarrollo del proyecto, se obtuvo como resultado un addins llamado MonoDevelop.SubscripionServices_1.0.mpack el cual puede ser descargado desde la página: http://addins.monodevelop.com/Project/SourceTagPackage/346?platform=Linux. Para instalarlo se emplea el Add-in Manager del MonoDevelop 2.8 con lo cual las opciones quedan instaladas dentro del menú Project del entorno a como se puede ver en la Figura 5.

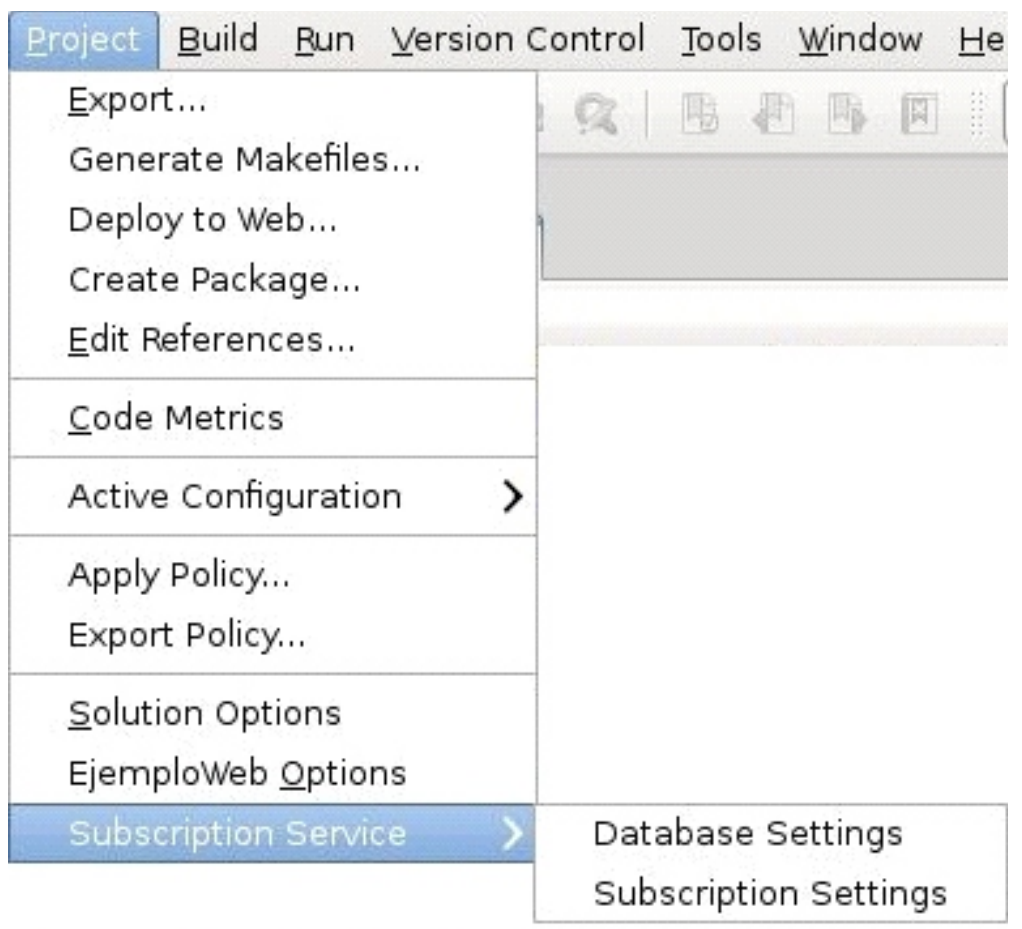

Figura 5. Visualización del addin desde el menú Project de MonoDevelop 2.8

Se logró que la primera opción del addins permitiera al usuario la configuración de la base de datos a través de la introducción de los valores de la base de datos a emplear, mientras que la segunda opción carga un sitio web el cual ha sido desarrollado con una interfaz similar a la empleada en Visual Studio 2008 para que el usuario se sienta familiarizado con la aplicación. 


\section{6- CONCLUSIONES}

La posibilidad de crear nuestros propios proveedores para el servicio de suscripciones de ASP.NET permite que esta funcionalidad pueda ser implementada sobre casi cualquier sistema gestor de bases de datos no teniendo que limitarnos a SQL Server que es el gestor por defecto.

La tecnología Mono.Addins con la cual ha sido desarrollado el IDE MonoDevelop 2.8 y la API de este, permitió desarrollar un addins que proveyese de interfaces para la gestión del servicio de subscripciones de una manera sencilla y a su vez, la compatibilidad de la plataforma Mono con la plataforma .NET permitió el desarrollo de una interfaz web, similar a la empleada en Visual Studio 2008 para la administración de sitios web.

\section{LÍNEAS DE TRABAJOS FUTUROS}

Del presente trabajo se desprenden las siguientes ideas para futuros:

Mejorar el addin para que este pueda funcionar con más gestores de base de datos y no solo con PostgreSQL 8.4. Agregar a la interfaz web soporte para múltiples idiomas.

Agregar al sitio web, una sección que permita confirmar las cuentas de usuario a través de email.

\section{7- AGRADECIMIENTOS}

El autor de este trabajo agradece profundamente al Ph.D. Francisco Javier Ceballos Sierra profesor titular de la Universidad de Alcalá de Henares, España por proponer la idea para la realización de este trabajo. 


\section{8- REFERENCIAS}

1. Ceballos, F. J. (2008). Aplicaciones .NET multiplataforma. Madrid, 1ra Ed, RA-MA Editorial, 212 pags.

2. Ceballos, F. J. (2009). Enciclopedia de Microsoft Visual C\#. Madrid, 3ra Ed, RA-MA Editorial, 1112 pags.

3. Jacobson, I., Booch, G., \& Rumbaugh, J. (2000). El proceso unificado de desarrollo de software. Madrid, 1ra Ed, Pearson Educación, S.A, 464 pags.

4. MonoDevelop. Addin development basics. [Sitio en Internet]. Consultado el 3 de Noviembre de 2011. Disponible en: http://monodevelop.com/Developers/Articles/Addin_development_basics

5. PostgreSQL. About PostgreSQL. [Sitio en Internet]. Consultado el 20 de Noviembre de 2011. Disponible en: http://www.postgresql.org/about/

6. Lluis, API Overview. [Sitio en Internet]. Consultado el 13 de Noviembre de 2011. Disponible en: http://monodevelop.com/Developers/Articles/API_Overview

7. MSDN. Managing Authorization Using Roles. [Sitio en Internet]. Consultado el 13 de Noviembre de 2011. Disponible en: http://msdn.microsoft.com/en-us/library/9ab2fxh0.aspx

8. MSDN. Managing Users by Using Membership. [Sitio en Internet]. Consultado el 2 de Noviembre de 2011. Disponible en: http://msdn.microsoft.com/en-us/library/tw292whz.aspx

9. Web Ref. Membership providers for MySQL. [Sitio en Internet]. Consultado el 24 de Enero de 2011. Disponible en: http://www.webref.eu/asp-net-security-mysqlmembershipprovider.php

10. Web Ref. Role providers for MySQL. [Sitio en Internet]. Consultado el 25 de Enero de 2011. Disponible en: http://www.webref.eu/asp-net-security-mysqlroleprovider.ph 